\title{
Nutrient intake among Chinese women living in Shanghai, China
}

\author{
Zhi Chen ${ }^{1}$, Xiao Ou Shu ${ }^{*}$, Gong Yang ${ }^{1}$, Honglan $\mathrm{Li}^{2}$, Qi $\mathrm{Li}^{2}$, Yu-Tang $\mathrm{Gao}^{2}$ and Wei Zheng ${ }^{1}$ \\ ${ }^{1}$ Department of Medicine, Center for Health Services Research and Vanderbilt-Ingram Cancer Center, Vanderbilt University, \\ Nashville, TN 37232-8300, USA \\ ${ }^{2}$ Department of Epidemiology, Shanghai Cancer Institute, Shanghai, People's Republic of China
}

(Received 11 October 2005 - Revised 30 March 2006 - Accepted 5 April 2006)

\begin{abstract}
It has been increasingly recognized that dietary factors play a major role in the development of chronic diseases, including cancers and CVD. The identification of patterns of nutrient intake in populations with different disease incidence will be helpful in understanding the diet and disease association. The present report describes nutrient intake in 74810 Chinese women, aged between 40 and 70 years, who participated in a population-based cohort study in Shanghai from 1997 to 2000. A food frequency questionnaire was used to derive estimates of nutrient intakes. The average daily energy intake was $7027.8 \mathrm{~kJ}$ in the study population, with protein, fat and carbohydrates contributing $15.9 \%$, $15.6 \%$ and $68.5 \%$, respectively. Factors, including younger age, higher income, attainment of education at the college level or above, being married or holding a professional job, were related to higher intake levels of most nutrients. The present results highlight the need for continuing to promote public health strategies aimed at improving the diets of women from both older and lower socio-demographic backgrounds, and in the meantime, continuing to help address the current dearth of data on nutrient intakes for middle-aged and elderly urban Chinese women.
\end{abstract}

Nutrient intake: Chinese urban women: Population sample

Nutrient factors have been linked with several chronic diseases experienced by women in their middle and later years, including CVD, some types of cancer, non-insulin-dependent diabetes and obesity-related diseases. The risk of these chronic health conditions increases with age, which can be at least partly attributed to nutritional factors (Holmes et al. 1999; Stampfer et al. 2000; Hu et al. 2001; Fung et al. 2004, 2005; Lee et al. 2005; Montonen et al. 2005). The nutrient status of women in this age group is of considerable public health interest. The rapid economic advancement that has taken place in China over the last 20 years has been accompanied by changes in diet. For example, the intake of animal-source foods has increased $50-160 \%$ in the last two decades with changes being much higher in Shanghai than in China overall (Lu \& Xiu, 1987; Yu et al. 1991; Popkin, 2003; Smith, 1993). Simultaneously, the incidence of cancer, obesity, hypertension, diabetes and CVD has also increased (Wu et al. 2001; Cheng, 2003; Wang et al. 2003; Yang et al. 2003). It is necessary to study nutrient and diet intake in women in order to monitor the potential health impacts of these changes. Although the number of nutrition studies in China has been increasing, few reports are available on middle-aged and elderly women living in urban Shanghai, where rapid development is occurring.

In 1997, we initiated a large population-based cohort study of 74942 women, the Shanghai Women's Health Study (SWHS), for a long-term aetiological study of cancer and other major chronic diseases. One of the primary foci of the SWHS was to identify dietary factors that may be related to the risk of cancer and other chronic diseases. The present paper describes the nutrient intake among this cohort of women overall and by socio-demographic factors and provides some baseline data for further in-depth studies of the health status of elderly Chinese women.

\section{Materials and methods}

The SWHS is a population-based cohort study of women aged 40-70 years. Detailed information on this cohort study has been described elsewhere (Zheng et al. 2005). Briefly, study recruitment was carried out from March 1997 to May 2000. Eligible participants were permanent female residents aged 40-70 years from seven communities in urban Shanghai. Of the 81170 eligible women who lived in the study communities at the time of the baseline survey, 75221 women participated in the study, with a participation rate of $92.7 \%$. Of those who completed the survey, 279 women were later found to be younger than 40 or older than 70 years at the time of the baseline interview and thus were excluded from the cohort. The remaining 74942 women constitute the SWHS. Of the 5949 non-participants, 2407 refused to participate (3.0\%), 2073 were absent during the study period $(2.6 \%)$ and 1469 $(1.8 \%)$ were excluded for other miscellaneous reasons. Also, we excluded from the current analysis 132 women whose

\footnotetext{
Abbreviations: FFQ, food frequency questionnaire; 24-HDR, 24 h dietary recalls; SWHS, Shanghai Women's Health Study.

* Corresponding author: Dr Xiao-Ou Shu, Vanderbilt Center for Health Services Research, Medical Center East, Suite 6000, 1215 21st Avenue South, Nashville, TN 37232-8300, USA, fax +1 615936 1269, email xiao-ou.shu@vanderbilt.edu
} 
average energy intake was less than $2090 \mathrm{~kJ} / \mathrm{d}$ or more than $14630 \mathrm{~kJ} / \mathrm{d}$

Dietary information was collected by interviewers using a validated food frequency questionnaire (FFQ), which was developed based on a similar dietary questionnaire used in several previous epidemiological studies of cancer in Shanghai (Shu et al. 1989, 1993; Zheng et al. 1992; Ji et al. 1998; Dai et al. 2001). The validity of the FFQ used in the present study has been evaluated (Shu et al. 2004). For a dietary calibration study, 200 SWHS participants were recruited. Study participants completed an FFQ at baseline and $24 \mathrm{~h}$ dietary recalls (24-HDR) twice per month consecutively for 12 months. At the end of the study, a second FFQ was administered. The FFQ included the foods that accounted for $86 \%$ of the foods recorded in the 24-HDR surveys. Validity of the FFQ was evaluated by comparing intake levels of major nutrients and foods obtained from the second FFQ with those derived from the multiple 24-HDR. The median intake for major nutrients from the second FFQ and the 24-HDR was similar, with the differences ranging from 1.3 to $12 \cdot 1 \%$. Correlation coefficients for nutrients assessed by the FFQ and the multiple 24-HDR were 0.59-0.66 for macronutrients and 0.41-0.59 for micronutrients.

The FFQ covered about $90 \%$ of the commonly consumed foods in urban Shanghai. For each food item or food group, subjects were asked how frequently (daily, weekly, monthly, yearly or never) they consumed the food or food groups during the past 12 months followed by a question on the amount consumed in liang ( 1 liang $=50 \mathrm{~g})$ per unit of time. For example, when study participants were queried regarding consumption of fish, they were first asked how often they ate fish (daily, weekly, monthly, yearly or never). If a participant ate fish on a weekly basis, she would be asked how many liangs of fish she ate each week. For seasonal foods (mainly vegetables and fruits), an in-season consumption pattern was elicited. During the baseline survey, approximately 1000 SWHS participants were randomly selected in each season and information was obtained from them on the number of months per year each food was consumed. The information was then regressed on the age, education and income of the subgroup of study participants. The regression coefficients were used as the weight to adjust for the variation of seasonal foods for the whole cohort of women, including the participants of the calibration study (Shu et al. 2004). The Chinese Food Composition Table (Wang and Shen 1991) was used to estimate the intake level of major nutrients for study participants. Total dietary intake of each nutrient was calculated by adding the intakes of that nutrient from the information collected for each food item. Nutrient intake for individual food items was estimated by multiplying the nutrient content listed in the Chinese Food Composition Table with the reported frequency and the amount of intake. In the study population $2 \%$ of women drank alcohol regularly, at an average of 0.6 drinks/d. Because of the low alcohol consumption rate, we did not include alcohol consumption in the nutrient intake estimation in this analysis.

Family income was divided into low, middle and high levels, corresponding to annual per capita income of less than $¥ 10000$, $¥ 10000-20000$ and more than $¥ 20000$. Occupation referred to participants' employment at study enrolment. 'Professional' referred to researchers, managers and other white-collar workers; 'manual workers' referred to blue-collar workers; and 'clerical' referred to people who provide service or supporting work. Marriage status was divided into two categories: currently married and living with husband, and currently unmarried, separated/divorced or widowed. We also compared the women aged 40-59 years in the present study with their counterparts in other countries. Data are mainly reported as means and standard deviations. Comparisons of nutrient intake among subjects with different socio-economic factors were carried out using the ANOVA test. Differences in nutrient intake were regarded as significant when $P<0 \cdot 05$. All analyses were performed using the programs provided by SAS (SAS Institute Inc., Cary, NC, USA).

\section{Results}

Table 1 shows the socio-demographic characteristics of the study participants by age groups. The mean age of women in the cohort was 52.1 years. Approximately $42 \%$ of women attained a high school education or higher, about $37 \%$ attended middle school and $21.6 \%$ had attended only elementary school or had no formal education. Nearly all women had worked outside the home. Approximately $40 \%$ of women at the study recruitment worked as manual labourers, and $36 \%$ of women were in professional occupations. There were 708 women $(3.76 \%)$ still working at the time of interview who were in the oldest category $(61-70$ years $)$ in the study. The vast majority of study participants $(89 \%)$ were married and living with a spouse at the time of study enrolment. Average BMI increased with age, which was 23.34, 24.45 and 25.02 for age groups 40-50, 51-60 and 61-70 years, respectively.

Table 2 presents the average daily nutrient intakes for all participants and for participants separated by age groups. Medians of these nutrient intakes were similar to the mean levels. Plant-based foods accounted for $82.7 \%$ of energy, $62.2 \%$ protein and $39.4 \%$ fat intake in the study. Rice and wheat flour were the main sources of carbohydrates for these Chinese women and accounted for $79.3 \%$ of carbohydrate intake in the present study population. Daily intakes of macro- and micronutrients in older women (aged 61-70 years) were lower than those of younger women. There was an inverse association between the percentage of energy intake from fat and protein and the age of study participants: the younger the age, the higher the percentage of intake from fat and protein. In general, nutrient intake had a positive association with family income (Table 3) and educational attainment level (Table 4), with the exception of carbohydrates.

We also analysed nutrient data by marital status and occupation (data not shown in tables). Both married women and unmarried women had similar mean intakes of carbohydrates, total vitamin $A$, and $\beta-, \gamma$ - and $\delta$-tocopherol, while married women had higher intake levels of the other nutrients under study. The professional workers reported higher intakes of all nutrients except for carbohydrates, while manual labourers had the lowest intake levels for all nutrients. The nutrient intakes of clerical workers were between those of professional workers and manual labourers. The present results remained unchanged when the data were analysed based on nutrient density. 
Table 1. Socio-demographic characteristics of participants in the Shanghai Women's Health Study

\begin{tabular}{|c|c|c|c|c|c|c|}
\hline \multirow[b]{2}{*}{ Group } & \multicolumn{2}{|c|}{$40-50$ years $(n 38803)$} & \multicolumn{2}{|c|}{$51-60$ years $(n 17193)$} & \multicolumn{2}{|c|}{$61-70$ years $(n 18814)$} \\
\hline & $n$ & $\%$ & $n$ & $\%$ & $n$ & $\%$ \\
\hline \multicolumn{7}{|l|}{ Education } \\
\hline None/elementary school only & 972 & $2 \cdot 50$ & 3067 & $17 \cdot 84$ & 12111 & $64 \cdot 37$ \\
\hline Middle school & 20084 & $51 \cdot 76$ & 4674 & $27 \cdot 19$ & 2872 & $15 \cdot 27$ \\
\hline High & 13075 & 33.70 & 5612 & 32.64 & 2166 & 11.51 \\
\hline College and above & 4670 & $12 \cdot 04$ & 3837 & $22 \cdot 32$ & 1657 & $8 \cdot 81$ \\
\hline Missing & 2 & 0.01 & 3 & 0.02 & 8 & 0.04 \\
\hline \multicolumn{7}{|l|}{ Family income* } \\
\hline Low & 8069 & $20 \cdot 79$ & 4097 & 23.83 & 8599 & $45 \cdot 71$ \\
\hline Middle & 15264 & $39 \cdot 34$ & 6276 & $36 \cdot 50$ & 7536 & $40 \cdot 06$ \\
\hline High & 15461 & 39.84 & 6817 & 39.65 & 2675 & $14 \cdot 22$ \\
\hline Missing & 9 & 0.02 & 3 & 0.02 & 4 & 0.02 \\
\hline \multicolumn{7}{|l|}{ Occupation† } \\
\hline Retired & 8854 & $22 \cdot 82$ & 10654 & 61.97 & 17076 & $90 \cdot 76$ \\
\hline Professional & 9212 & $23 \cdot 74$ & 3964 & 23.06 & 669 & 3.56 \\
\hline Clerical & 7508 & $19 \cdot 35$ & 994 & $5 \cdot 78$ & 361 & 1.92 \\
\hline Manual workers & 13229 & 34.09 & 1581 & $9 \cdot 20$ & 708 & 3.76 \\
\hline \multicolumn{7}{|l|}{ Marital status $\ddagger$} \\
\hline Married & 36245 & 93.41 & 15593 & $90 \cdot 69$ & 14554 & $77 \cdot 36$ \\
\hline \multirow[t]{2}{*}{ Unmarried } & 2558 & 6.59 & 1600 & $9 \cdot 31$ & 4260 & $22 \cdot 64$ \\
\hline & Mean & SD & Mean & SD & Mean & SD \\
\hline Height (m) & 1.59 & 0.05 & 1.57 & 0.05 & 1.54 & 0.05 \\
\hline Weight (kg) & $59 \cdot 3$ & $8 \cdot 5$ & $60 \cdot 2$ & $9 \cdot 1$ & $59 \cdot 7$ & $9 \cdot 5$ \\
\hline BMI $\left(\mathrm{kg} / \mathrm{m}^{2}\right)$ & $23 \cdot 34$ & $3 \cdot 12$ & 24.45 & 3.45 & $25 \cdot 02$ & $3 \cdot 71$ \\
\hline
\end{tabular}

* Family income was divided into low, middle and high levels, corresponding to annual per capita income of less than $¥ 10000$, $¥ 10000$-20000 and more than $¥ 20000$.

†Occupation refers to participants' employment at study enrolment. 'Professional' refers to researchers, managers and other white-collar workers; 'manual workers' refers to blue-collar workers; and 'clerical' refers to people who provide service or supporting work.

$\ddagger$ Marital status has two categories: currently married and living with husband, and currently unmarried, separated/divorced or widowed.

Table 2. Average daily nutrient intakes by age, Shanghai Women's Health Study*

\begin{tabular}{|c|c|c|c|c|c|c|c|c|c|}
\hline \multirow[b]{2}{*}{ Nutrients } & \multicolumn{3}{|c|}{ Total $(n 74810)$} & \multicolumn{2}{|c|}{$\begin{array}{c}40-50 \text { years } \\
(n 38803)\end{array}$} & \multicolumn{2}{|c|}{$\begin{array}{c}51-60 \text { years } \\
(n 17193)\end{array}$} & \multicolumn{2}{|c|}{$\begin{array}{c}61-70 \text { years } \\
(n 18814)\end{array}$} \\
\hline & Mean & SD & Median & Mean & SD & Mean & SD & Mean & SD \\
\hline Energy (kJ) & $7027 \cdot 8$ & $1654 \cdot 0$ & $6862 \cdot 7$ & $7119 \cdot 8$ & $1644 \cdot 0$ & $7167 \cdot 4$ & $1616 \cdot 7$ & 6711.0 & $1646 \cdot 1$ \\
\hline Protein (g) & $67 \cdot 1$ & $20 \cdot 7$ & 64.5 & $69 \cdot 3$ & $20 \cdot 6$ & $68 \cdot 2$ & $20 \cdot 4$ & $61 \cdot 7$ & $20 \cdot 3$ \\
\hline Fat (g) & $29 \cdot 6$ & $13 \cdot 2$ & $27 \cdot 6$ & $31 \cdot 2$ & $13 \cdot 3$ & $29 \cdot 7$ & $12 \cdot 8$ & $26 \cdot 3$ & $12 \cdot 7$ \\
\hline Carbohydrate (g) & $286 \cdot 6$ & $67 \cdot 2$ & $279 \cdot 8$ & $286 \cdot 3$ & $65 \cdot 9$ & $293 \cdot 8$ & $68 \cdot 0$ & $280 \cdot 6$ & $68 \cdot 3$ \\
\hline \multicolumn{10}{|l|}{ Percentage of energy from } \\
\hline Protein & $15 \cdot 9$ & $2 \cdot 6$ & & $16 \cdot 2$ & $2 \cdot 6$ & $15 \cdot 8$ & $2 \cdot 6$ & $15 \cdot 3$ & $2 \cdot 7$ \\
\hline Fat & $15 \cdot 6$ & $4 \cdot 8$ & & $16 \cdot 3$ & $4 \cdot 7$ & $15 \cdot 3$ & $4 \cdot 7$ & $14 \cdot 4$ & 4.9 \\
\hline Carbohydrate & 68.5 & $6 \cdot 8$ & & $67 \cdot 6$ & $6 \cdot 6$ & $68 \cdot 8$ & $6 \cdot 7$ & $70 \cdot 3$ & $7 \cdot 0$ \\
\hline Insoluble fibre (g) & $10 \cdot 6$ & $4 \cdot 1$ & $10 \cdot 4$ & $10 \cdot 8$ & $4 \cdot 0$ & $10 \cdot 9$ & $4 \cdot 1$ & $10 \cdot 0$ & $4 \cdot 1$ \\
\hline Thiamin (mg) & 0.9 & 0.3 & 0.9 & 1.0 & 0.3 & 0.9 & 0.3 & 0.9 & 0.3 \\
\hline Riboflavin (mg) & 0.9 & 0.3 & $0 \cdot 8$ & 0.9 & 0.3 & 0.9 & 0.3 & 0.8 & 0.3 \\
\hline Ascorbic acid (mg) & 93.6 & $50 \cdot 3$ & 83.9 & $98 \cdot 7$ & $51 \cdot 1$ & $95 \cdot 2$ & $50 \cdot 2$ & $81 \cdot 4$ & $46 \cdot 7$ \\
\hline Niacin (mg) & $14 \cdot 3$ & $4 \cdot 3$ & $13 \cdot 7$ & 14.9 & $4 \cdot 3$ & $14 \cdot 6$ & $4 \cdot 2$ & $13 \cdot 1$ & $4 \cdot 1$ \\
\hline Total vitamin A $(\mu \mathrm{g})$ & $627 \cdot 8$ & $320 \cdot 2$ & $572 \cdot 1$ & $665 \cdot 6$ & $328 \cdot 7$ & $627 \cdot 6$ & $310 \cdot 4$ & $550 \cdot 2$ & $296 \cdot 4$ \\
\hline Retinol $(\mu \mathrm{g})$ & $188 \cdot 6$ & 174.5 & $154 \cdot 1$ & $214 \cdot 4$ & $192 \cdot 8$ & $179 \cdot 7$ & $156 \cdot 9$ & $143 \cdot 6$ & 135.9 \\
\hline Carotene $(\mu \mathrm{g})$ & $2709 \cdot 6$ & $1488 \cdot 2$ & $2432 \cdot 5$ & $2786 \cdot 3$ & 1474.9 & $2758 \cdot 1$ & $1499 \cdot 5$ & $2507 \cdot 0$ & $1486 \cdot 5$ \\
\hline Total vitamin E (mg) & $13 \cdot 3$ & $6 \cdot 0$ & $12 \cdot 3$ & $13 \cdot 5$ & $5 \cdot 8$ & $13 \cdot 7$ & $6 \cdot 1$ & $12 \cdot 5$ & $6 \cdot 1$ \\
\hline$\alpha$-Tocopherol (mg) & $4 \cdot 2$ & $1 \cdot 8$ & $4 \cdot 0$ & 4.5 & $1 \cdot 8$ & $4 \cdot 2$ & $1 \cdot 8$ & 3.6 & $1 \cdot 7$ \\
\hline$\beta$ - and $\gamma$-Tocopherol (mg) & $4 \cdot 2$ & 2.5 & $3 \cdot 7$ & $4 \cdot 2$ & 2.4 & $4 \cdot 4$ & $2 \cdot 6$ & $4 \cdot 1$ & $2 \cdot 6$ \\
\hline$\delta$-Tocopherol (mg) & 3.6 & $2 \cdot 3$ & $3 \cdot 1$ & 3.5 & $2 \cdot 1$ & $3 \cdot 8$ & $2 \cdot 4$ & 3.5 & 2.4 \\
\hline Calcium (mg) & $463 \cdot 9$ & $201 \cdot 0$ & $438 \cdot 1$ & $473 \cdot 2$ & $196 \cdot 2$ & $479 \cdot 9$ & $204 \cdot 5$ & $430 \cdot 0$ & 203.5 \\
\hline Iron (mg) & $18 \cdot 6$ & $5 \cdot 3$ & $17 \cdot 9$ & $19 \cdot 0$ & $5 \cdot 3$ & $19 \cdot 0$ & $5 \cdot 3$ & $17 \cdot 5$ & $5 \cdot 3$ \\
\hline Haem iron (mg) & $3 \cdot 2$ & 1.8 & 2.9 & 3.5 & $1 \cdot 8$ & $3 \cdot 1$ & 1.7 & 2.5 & $2 \cdot 2$ \\
\hline Potassium (mg) & $1789 \cdot 8$ & $644 \cdot 1$ & $1702 \cdot 0$ & $1859 \cdot 2$ & $636 \cdot 1$ & $1830 \cdot 6$ & $644 \cdot 8$ & $1609 \cdot 1$ & 625.4 \\
\hline Magnesium (mg) & 283.4 & $82 \cdot 8$ & $272 \cdot 6$ & $288 \cdot 6$ & $81 \cdot 4$ & $290 \cdot 5$ & $83 \cdot 1$ & $266 \cdot 1$ & $83 \cdot 0$ \\
\hline Sodium (mg) & $340 \cdot 9$ & $151 \cdot 9$ & 318.9 & $364 \cdot 6$ & $152 \cdot 6$ & 341.6 & $148 \cdot 8$ & 291.4 & $141 \cdot 3$ \\
\hline Phosphorus (mg) & $955 \cdot 6$ & $280 \cdot 0$ & 923.4 & 978.5 & $176 \cdot 5$ & $975 \cdot 0$ & $278 \cdot 2$ & $890 \cdot 6$ & 278.9 \\
\hline Selenium $(\mu \mathrm{g})$ & $45 \cdot 0$ & $21 \cdot 3$ & 41.0 & $48 \cdot 7$ & 21.9 & $44 \cdot 7$ & $20 \cdot 4$ & $37 \cdot 6$ & $18 \cdot 8$ \\
\hline
\end{tabular}

*All $P$ values for differences between the subgroup nutrients were $<0.001$. 
Table 3. Average daily nutrient intakes by income, Shanghai Women's Health Study*

\begin{tabular}{|c|c|c|c|c|c|c|}
\hline \multirow[b]{2}{*}{ Nutrients } & \multicolumn{2}{|c|}{ Low (n 20765) } & \multicolumn{2}{|c|}{ Middle ( $n 29$ 076) } & \multicolumn{2}{|c|}{ High (n 24 953) } \\
\hline & Mean & SD & Mean & SD & Mean & SD \\
\hline Energy (kJ) & 6884.9 & $1729 \cdot 3$ & 7057.5 & $1643 \cdot 6$ & $7112 \cdot 7$ & $1593 \cdot 8$ \\
\hline Protein (g) & $63 \cdot 3$ & $20 \cdot 9$ & $67 \cdot 2$ & $20 \cdot 4$ & $70 \cdot 2$ & $20 \cdot 3$ \\
\hline Fat $(\mathrm{g})$ & $27 \cdot 3$ & $13 \cdot 7$ & $29 \cdot 5$ & $12 \cdot 9$ & $31 \cdot 7$ & $12 \cdot 7$ \\
\hline Carbohydrate (g) & $287 \cdot 1$ & $70 \cdot 9$ & $288 \cdot 6$ & $67 \cdot 2$ & 283.9 & $63 \cdot 8$ \\
\hline \multicolumn{7}{|l|}{ Percentage of energy from } \\
\hline Protein & $15 \cdot 3$ & $2 \cdot 6$ & $15 \cdot 8$ & $2 \cdot 6$ & $16 \cdot 4$ & $2 \cdot 5$ \\
\hline Fat & 14.6 & $5 \cdot 0$ & $15 \cdot 5$ & $4 \cdot 7$ & $16 \cdot 6$ & $4 \cdot 6$ \\
\hline Carbohydrate & $70 \cdot 2$ & $7 \cdot 1$ & $68 \cdot 7$ & $6 \cdot 7$ & $67 \cdot 0$ & $6 \cdot 5$ \\
\hline Insoluble fibre (g) & $10 \cdot 1$ & $4 \cdot 1$ & $10 \cdot 6$ & $4 \cdot 0$ & $11 \cdot 1$ & $4 \cdot 0$ \\
\hline Thiamin (mg) & 0.9 & $0 \cdot 3$ & 0.9 & 0.3 & $1 \cdot 0$ & 0.3 \\
\hline Riboflavin (mg) & 0.8 & 0.3 & 0.9 & 0.3 & $1 \cdot 0$ & 0.3 \\
\hline Ascorbic acid (mg) & $85 \cdot 6$ & $49 \cdot 9$ & $93 \cdot 3$ & $49 \cdot 8$ & $100 \cdot 5$ & $50 \cdot 4$ \\
\hline Niacin (mg) & $13 \cdot 6$ & $4 \cdot 3$ & $14 \cdot 4$ & $4 \cdot 2$ & $14 \cdot 9$ & $4 \cdot 2$ \\
\hline Total vitamin A $(\mu \mathrm{g})$ & $582 \cdot 2$ & $327 \cdot 8$ & $628 \cdot 0$ & $316 \cdot 3$ & $665 \cdot 7$ & $313 \cdot 4$ \\
\hline Retinol $(\mu \mathrm{g})$ & $166 \cdot 3$ & $176 \cdot 1$ & $186 \cdot 7$ & $173 \cdot 2$ & $209 \cdot 4$ & $172 \cdot 3$ \\
\hline Carotene $(\mu \mathrm{g})$ & $2568 \cdot 3$ & $1534 \cdot 2$ & $2723 \cdot 7$ & 1478.5 & 2810.9 & $1451 \cdot 2$ \\
\hline Total vitamin E (mg) & $12 \cdot 6$ & $6 \cdot 1$ & $13 \cdot 3$ & 5.9 & $13 \cdot 9$ & $5 \cdot 8$ \\
\hline$\alpha$-Tocopherol (mg) & $3 \cdot 8$ & $1 \cdot 8$ & $4 \cdot 2$ & $1 \cdot 8$ & $4 \cdot 6$ & $1 \cdot 8$ \\
\hline$\beta$ - and $\gamma$-Tocopherol (mg) & $4 \cdot 0$ & $2 \cdot 6$ & $4 \cdot 2$ & $2 \cdot 5$ & 4.4 & $2 \cdot 4$ \\
\hline$\delta$-Tocopherol (mg) & $3 \cdot 4$ & $2 \cdot 4$ & $3 \cdot 6$ & $2 \cdot 3$ & $3 \cdot 7$ & $2 \cdot 2$ \\
\hline Calcium (mg) & $423 \cdot 0$ & $199 \cdot 2$ & $460 \cdot 3$ & $197 \cdot 3$ & $502 \cdot 1$ & $199 \cdot 5$ \\
\hline Iron (mg) & $17 \cdot 9$ & $5 \cdot 5$ & $18 \cdot 7$ & $5 \cdot 3$ & $19 \cdot 1$ & $5 \cdot 2$ \\
\hline Haem iron (mg) & $2 \cdot 7$ & $1 \cdot 8$ & $3 \cdot 1$ & 1.8 & $3 \cdot 6$ & $1 \cdot 8$ \\
\hline Potassium (mg) & $1647 \cdot 7$ & $641 \cdot 0$ & $1783 \cdot 6$ & $631 \cdot 3$ & $1915 \cdot 3$ & $636 \cdot 1$ \\
\hline Magnesium (mg) & $270 \cdot 4$ & $84 \cdot 0$ & 283.9 & $82 \cdot 0$ & 293.6 & $81 \cdot 2$ \\
\hline Sodium (mg) & $302 \cdot 8$ & $147 \cdot 7$ & $337 \cdot 1$ & $148 \cdot 0$ & $377 \cdot 1$ & $151 \cdot 7$ \\
\hline Phosphorus (mg) & $904 \cdot 4$ & 283.4 & 955.9 & 276.5 & $997 \cdot 8$ & $274 \cdot 2$ \\
\hline Selenium $(\mu \mathrm{g})$ & $39 \cdot 8$ & $20 \cdot 2$ & $44 \cdot 6$ & $20 \cdot 8$ & $49 \cdot 8$ & $21 \cdot 8$ \\
\hline
\end{tabular}

\section{Discussion}

The observed trend of decreasing intake of most nutrients in elderly women compared to the other adult groups in the present study is supported by previous studies (Ryan et al. 1992; Wright et al. 1995). The reduction in energy intake with age is compatible with the reduction in energy requirements experienced as a result of ageing. Energy requirements lessen as a consequence of reduced BMR due to the loss of fat-free mass and reduction in physical activity. With a reduction in energy intake, the intake of many micronutrients is consequently expected to decrease as well (Willett, 1998). The official retirement age in China is 50-55 years old for female clerical or blue-collar workers, 60 years for female professional or government employees. In the present study population, only a few women still worked, and $91 \%$ of women aged between 60 and 70 years were retired at study recruitment. Decreased food intake, a sedentary lifestyle and reduced energy expenditure in older women together are risk factors that contribute to the decline of bodily functions and the development of a number of age-related diseases (Meydani, 2001).

The results of the present study show large socio-economic variations in nutrient intake among middle aged and elderly Chinese women in Shanghai. Women with lower education levels, lower income levels and with a non-professional occupation tended to have a lower intake of macro- and micronutrients than those with higher education levels, higher income and those working as professionals. The present results agree with literature reporting that subjects with low socio-economic status have lower and less varied intake of fruits and vegetables and lower intake of many micronutrients than those with higher socio-economic status (Baghurst et al. 1994; Davey Smith \& Brunner, 1997; Dobson et al. 1997; De IralaEstevez et al. 2000; Giskes et al. 2002; Mishra et al. 2002). People with lower education levels were generally employed in labour or low-skilled occupations in China, and they had lower income. This emphasizes the importance of considering these aspects in combination, rather than focusing on one factor at a time when researching how Chinese women can improve their diet.

The present study showed that married women had higher intakes of micro- and macronutrients than unmarried women, which is consistent with past reports that nutrients were less congruent among women who were single, divorced/separated, widowed or living alone, compared with those living with a partner (Billson et al. 1999; Woo et al. 1999).

We also compared women aged 40-59 years in the present study with their counterparts in an international study (Table 5). The international study was conducted among women aged 40-59 years from 1997 to 1999 and collected four standardized 24-HDR from each participant on two pairs of successive days about 3-6 weeks apart. No physical activity information was available in the international study (Zhou et al. 2003). There were marked differences in 
Table 4. Average daily nutrient intakes by education, Shanghai Women's Health Study*

\begin{tabular}{|c|c|c|c|c|c|c|c|c|}
\hline \multirow[b]{2}{*}{ Nutrients } & \multicolumn{2}{|c|}{$\begin{array}{l}\text { Under/elementary } \\
\quad(n 16150)\end{array}$} & \multicolumn{2}{|c|}{$\begin{array}{l}\text { Middle school } \\
\quad(n 27630)\end{array}$} & \multicolumn{2}{|c|}{$\begin{array}{l}\text { High school } \\
\text { (n 20 853) }\end{array}$} & \multicolumn{2}{|c|}{$\begin{array}{l}\text { College and above } \\
\qquad(n 10164)\end{array}$} \\
\hline & Mean & SD & Mean & SD & Mean & SD & Mean & SD \\
\hline Energy (kJ) & 6714.8 & $1702 \cdot 1$ & $7121 \cdot 0$ & $1670 \cdot 7$ & $7104 \cdot 3$ & $1626 \cdot 4$ & $7115 \cdot 6$ & $1520 \cdot 7$ \\
\hline Protein $(\mathrm{g})$ & 59.6 & $19 \cdot 7$ & $68 \cdot 0$ & $20 \cdot 4$ & 69.9 & $20 \cdot 8$ & $71 \cdot 2$ & $19 \cdot 8$ \\
\hline Fat (g) & $24 \cdot 6$ & $12 \cdot 4$ & $30 \cdot 1$ & $13 \cdot 3$ & $31 \cdot 5$ & $13 \cdot 0$ & $32 \cdot 4$ & $12 \cdot 2$ \\
\hline Carbohydrate (g) & $286 \cdot 7$ & $72 \cdot 5$ & $290 \cdot 3$ & $67 \cdot 8$ & $284 \cdot 2$ & 64.7 & 281.5 & $60 \cdot 7$ \\
\hline Protein & $14 \cdot 7$ & $2 \cdot 5$ & $15 \cdot 9$ & $2 \cdot 5$ & $16 \cdot 4$ & $2 \cdot 6$ & $16 \cdot 7$ & $2 \cdot 5$ \\
\hline Fat & $13 \cdot 5$ & 4.9 & $15 \cdot 6$ & $4 \cdot 8$ & $16 \cdot 4$ & $4 \cdot 6$ & $17 \cdot 0$ & $4 \cdot 4$ \\
\hline Carbohydrate & $71 \cdot 7$ & $6 \cdot 8$ & $68 \cdot 5$ & $6 \cdot 6$ & $67 \cdot 2$ & $6 \cdot 5$ & $66 \cdot 4$ & $6 \cdot 2$ \\
\hline Insoluble fibre (g) & $9 \cdot 7$ & $4 \cdot 0$ & $10 \cdot 7$ & $4 \cdot 0$ & $11 \cdot 0$ & $4 \cdot 1$ & $11 \cdot 1$ & $4 \cdot 0$ \\
\hline Thiamin (mg) & $0 \cdot 8$ & 0.3 & 0.9 & 0.3 & 0.9 & 0.3 & 1.0 & 0.3 \\
\hline Riboflavin (mg) & 0.7 & 0.3 & 0.9 & 0.3 & 0.9 & 0.3 & $1 \cdot 0$ & 0.3 \\
\hline Ascorbic acid (mg) & $78 \cdot 3$ & $46 \cdot 7$ & $95 \cdot 0$ & $49 \cdot 8$ & $100 \cdot 0$ & $51 \cdot 5$ & $100 \cdot 7$ & $49 \cdot 8$ \\
\hline Niacin (mg) & $12 \cdot 9$ & $4 \cdot 0$ & $14 \cdot 6$ & $4 \cdot 3$ & $14 \cdot 8$ & $4 \cdot 3$ & $15 \cdot 0$ & $4 \cdot 1$ \\
\hline Carotene $(\mu \mathrm{g})$ & $2444 \cdot 4$ & $1493 \cdot 4$ & 2734.6 & 1463.5 & $2826 \cdot 0$ & $1508 \cdot 7$ & $2825 \cdot 0$ & $1451 \cdot 7$ \\
\hline Total vitamin $\mathrm{E}(\mathrm{mg})$ & 11.9 & $6 \cdot 1$ & 13.4 & 5.9 & 13.9 & 5.9 & $14 \cdot 1$ & $5 \cdot 7$ \\
\hline$\alpha$-Tocopherol (mg) & $3 \cdot 4$ & $1 \cdot 7$ & $4 \cdot 3$ & $1 \cdot 8$ & $4 \cdot 6$ & $1 \cdot 8$ & $4 \cdot 7$ & $1 \cdot 7$ \\
\hline$\beta$ - and $\gamma$-Tocopherol (mg) & $3 \cdot 8$ & $2 \cdot 6$ & $4 \cdot 2$ & $2 \cdot 5$ & 4.4 & $2 \cdot 5$ & 4.5 & $2 \cdot 4$ \\
\hline$\delta$-Tocopherol (mg) & $3 \cdot 3$ & $2 \cdot 4$ & $3 \cdot 6$ & $2 \cdot 3$ & $3 \cdot 7$ & $2 \cdot 2$ & $3 \cdot 8$ & $2 \cdot 1$ \\
\hline Calcium (mg) & 393.8 & $191 \cdot 6$ & 455.4 & 193.4 & $497 \cdot 4$ & $201 \cdot 0$ & $529 \cdot 8$ & $199 \cdot 1$ \\
\hline Iron (mg) & $17 \cdot 2$ & $5 \cdot 3$ & $18 \cdot 8$ & $5 \cdot 3$ & $19 \cdot 1$ & $5 \cdot 4$ & $19 \cdot 2$ & $5 \cdot 1$ \\
\hline Haem iron (mg) & $2 \cdot 3$ & 1.5 & $3 \cdot 2$ & $1 \cdot 8$ & 3.5 & $1 \cdot 8$ & $3 \cdot 7$ & 1.7 \\
\hline Potassium (mg) & $1526 \cdot 6$ & $601 \cdot 3$ & $1803 \cdot 7$ & $627 \cdot 3$ & 1901.4 & $649 \cdot 0$ & 1941.5 & $620 \cdot 8$ \\
\hline Magnesium (mg) & $259 \cdot 0$ & 81.5 & 285.9 & $81 \cdot 7$ & $292 \cdot 9$ & $83 \cdot 2$ & $296 \cdot 1$ & $79 \cdot 1$ \\
\hline Sodium (mg) & $265 \cdot 7$ & $130 \cdot 1$ & $342 \cdot 2$ & $146 \cdot 6$ & 373.9 & $155 \cdot 1$ & $389 \cdot 1$ & $147 \cdot 1$ \\
\hline Phosphorus (mg) & 857.5 & $270 \cdot 0$ & $961 \cdot 7$ & $275 \cdot 4$ & 993.4 & $280 \cdot 0$ & $1017 \cdot 4$ & $268 \cdot 4$ \\
\hline Selenium $(\mu \mathrm{g})$ & 34.7 & $17 \cdot 2$ & $45 \cdot 9$ & $20 \cdot 8$ & $49 \cdot 4$ & 22.5 & $50 \cdot 0$ & $20 \cdot 8$ \\
\hline
\end{tabular}

*All $P$ values for differences between the subgroup nutrients were $<0.001$ with the exception of carbohydrates.

macronutrient consumption between Chinese women and women in other countries, reflecting the general differences in overall dietary patterns. The participants in the present study had the lowest average daily intakes of energy, calcium, retinol, vitamin A and phosphorus compared to participants in studies conducted in Japan, the UK and the USA. Total fat provided $16.1 \%, 26.1 \%, 32.5 \%$ and $32.6 \%$ of total calories for Chinese, Japanese, British and American women, respectively. The percentage of calories from protein was similar for women from Japan, the UK and China (16.1\%) and was a little lower for American women (15.6\%). Energy from carbohydrates, intake of vitamin $\mathrm{E}$, iron and magnesium were the

Table 5. Comparison of daily nutrient intake between women aged 40-59 years in four countries*

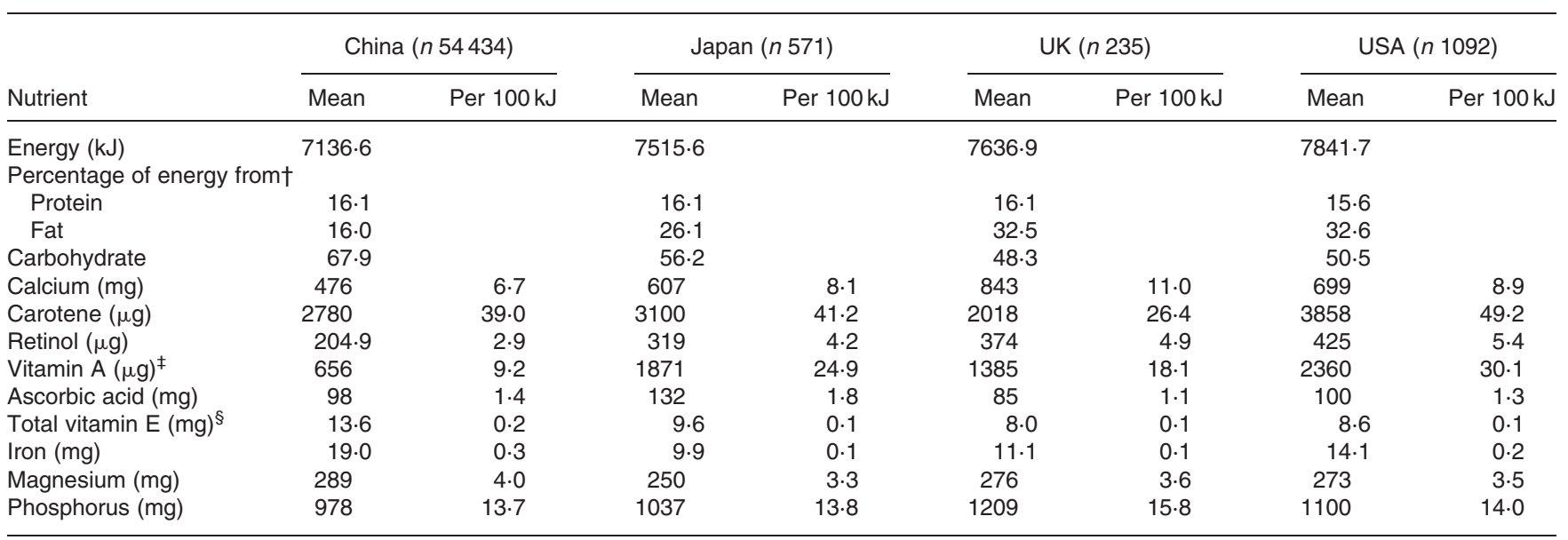

* Data for Japan, UK and USA are from the INTERMAP study (Zhou et al. 2003). The INTERMAP study was conducted from 1997 to 1999 and collected four standardized

$24 \mathrm{~h}$ dietary recalls from each participant on two pairs of successive days about $3-6$ weeks apart. No physical activity information was available in the study.

†The sum of energy from protein, fat and carbohydrate for Japan, UK and USA is less than $100 \%$, the rest of the energy comes from alcohol.

$¥$ The unit for vitamin $\mathrm{A}$ in original data was IU.

$\S$ Sum of $\alpha$-, $\beta$-, $\gamma$ - and $\delta$-tocopherol for Chinese, and $\alpha$-tocopherol equivalents for the other populations. 
highest among Chinese women relative to the other three countries. Intake of carotene in the present study in China was greater than in the UK, and less than in Japan and the USA. The level of consumption of ascorbic acid among Chinese women was higher than among British women and lower than consumption among Japanese and American women. Although a detailed comparison between studies is always difficult because of possible differences in sampling, dietary assessment methods and data-processing methods, it was clear that the composition of macro- and micronutrients in the nutrient intake of the Chinese women in the present study was quite different from the nutrient intake found in other countries. The difference between Chinese women and their counterparts may be related to different incidences of some chronic diseases, e.g. IHD. The age-standardized IHD mortality rates, per 100000 population, were $20 \cdot 8,47 \cdot 9,80 \cdot 7$ and 76.8 for Japanese, Chinese, British and American women, respectively (World Health Organization, 2006). Comparatively low intake of total protein and calcium for Chinese women may also be factors playing a role in the development of IHD.

The present study has many strengths. The large sample size meant that women from a wide range of socio-demographic backgrounds were included, making this one of only a few studies that has the ability to make these subgroup comparisons. Both the present and past findings of subgroup differences in macro- and micronutrient intakes have demonstrated the importance of ensuring that sample sizes and study design factors in large-scale surveys permit reliable comparisons across subgroups (Cook et al. 2001). A further strength of the present study is the comprehensive dietary assessment using a FFQ. In the study population, the foods supplied and dietary intake, especially for fruits and vegetables, varied with the seasons. FFQ, despite their well-documented limitations, are more useful than short-term dietary recall methods (e.g. 24-HDR) in representing individuals' overall dietary patterns (Cameron \& Van Staveren, 1988; Byers, 2001; Subar et al. 2001). The FFQ in the present study has been shown to provide a reliable and accurate method of measuring usual and habitual intake of nutrients among women in Shanghai (Cai et al. 2004; Shu et al. 2004).

The limitations of the present study should also be acknowledged. First, the nutrient data presented here relate only to middle-aged and elderly urban Chinese women. Also, dietary under-reporting is a widespread problem in self-reported dietary studies. In the present study, the mean of energy intake to BMR was $1.33,1.39$ and 1.36 for women of age groups 40-50, 51-60 and 61-70 years, respectively. In the age groups $40-50,51-60$ and $61-70$ years, $27.7 \%, 26.0 \%$ and $26.4 \%$ of women, respectively, were considered low-energy reporters (i.e. mean of energy intake to BMR was between 1.14 and 1.34). Furthermore, the use of an FFQ as the dietary assessment measure may have attenuated some of the impact of under-reporting typical on dietary studies, since FFQ do not attempt to measure the precise dietary intake of individuals. Last, a comparison of nutrient intake with dietary recommendations was not possible due to a lack of age-specific recommendations for nutrient intakes for Chinese women.

In summary, the present study provides important insights into the socio-economic variations of nutrient intake among middle-aged and elderly urban Chinese women. Chinese women have a better nutrient intake than their Western counterparts in terms of energy balance. From a public health viewpoint, the present findings of inadequate intakes of a range of nutrients, particularly among older women and women with lower socio-economic status, highlight the need for continuing to promote public health strategies aimed at improving the diets of women and of overcoming the adverse effects and maintaining the protective effects of nutrient intake.

\section{Acknowledgements}

This work was supported by USPHS grant no. R01CA70867 and research contracts from the National Cancer Institute.

\section{References}

Baghurst KI, Baghurst PA \& Record SJ (1994) Demographic and dietary profiles of high and low fat consumers in Australia. $J$ Epidemiol Community Health 48, 26-32.

Billson H, Pryer JA \& Nichols R (1999) Variation in fruit and vegetable consumption among adults in Britain: an analysis from the dietary and nutritional survey of British adults. Eur J Clin Nutr 53, 946-952.

Byers T (2001) Food frequency dietary assessment: how bad is good enough? Am J Epidemiol 154, 1087-1088.

Cai H, Shu XO, Hebert JR, Jin F, Yang G, Liu DK, Gao YT \& Zheng W (2004) Variation in nutrient intakes among women in Shanghai, China. Eur J Clin Nutr 58, 1604-1611.

Cameron ME \& Van Staveren WA (1988) Manual on Methodology for Food Consumption Studies. New York: Oxford University Press.

Cheng TO (2003) Rising prevalence of diabetes in China. J Natl Med Assoc 95, 1115-1116.

Cook T, Rutishauser IHE \& Seelig M (2001) Comparable Data on Food and Nutrient Intake and Physical Measurements from the 1983, 1985 and 1995 National Nutrition Surveys. Canberra: Commonwealth Department of Health and Aged Care.

Dai Q, Shu XO, Jin F, Kushi LH, Potter JD, Teas J, Ruan ZX, Gao YT \& Zheng W (2001) Association between soyfood intake and breast cancer risk by body mass index and estrogen receptor/progesterone receptor status. Br J Cancer 85, 372-378.

Davey Smith G \& Brunner E (1997) Socioeconomic differentials in health: the role of nutrition. Proc Nutr Soc 56, 75-90.

De Irala-Estevez J, Groth M, Johansson L, Oltersdorf U, Prattala R \& Martinez-Gonzalez MA (2000) A systematic review of socioeconomic differences in food habits in Europe: consumption of fruit and vegetables. Eur J Clin Nutr 54, 706-714.

Dobson A, Porteous J, McElduff P \& Alexander H (1997) Dietary trends: estimates from food supply and survey data. Eur J Clin Nutr 51, 193-198.

Fung TT, Hu FB, Holmes MD, Rosner BA, Hunter DJ, Colditz GA \& Willett WC (2005) Dietary patterns and the risk of postmenopausal breast cancer. Int J Cancer 116, 116-121.

Fung TT, Stampfer MJ, Manson JE, Rexrode KM, Willett WC \& Hu FB (2004) Prospective study of major dietary patterns and stroke risk in women. Stroke 35, 2014-2019.

Giskes K, Turrell G, Patterson C \& Newman B (2002) Socioeconomic differences among Australian adults in consumption of fruit and vegetables and intakes of vitamins A, C, and folate. J Hum Nutr Diet 15, 375-385.

Holmes MD, Hunter DJ, Colditz GA, Stampfer MJ, Hankinson SE, Speizer FE, Rosner B \& Willett WC (1999) Association of dietary 
intake of fat and fatty acids with risk of breast cancer. JAMA $\mathbf{2 8 1}$, 914-920.

Hu FB, Manson JE, Stampfer MJ, Colditz G, Liu S, Solomon CG \& Willett WC (2001) Diet, lifestyle, and the risk of type 2 diabetes mellitus in women. N Engl J Med 345, 790-797.

Ji BT, Chow WH, Yang G, McLaughlin JK, Zheng W, Shu XO, Jin F, Gao RN, Gao YT \& Fraumeni JF Jr (1998) Dietary habits and stomach cancer in Shanghai, China. Int J Cancer 76, 659-664.

Lee DH, Folsom AR \& Jacobs DR Jr (2005) Iron, zinc, and alcohol consumption and mortality from cardiovascular diseases: the Iowa Women's Health Study. Am J Clin Nutr 81, 787-791.

Lu RF \& Xiu DD (1987) A study of association between cancer incidence and diet in Shanghai. Tumor (Shanghai) 7, 68-70.

Meydani M (2001) Nutrition interventions in aging and age-associated disease. Ann N Y Acad Sci 928, 226-235.

Mishra G, Ball K, Arbuckle J \& Crawford D (2002) Dietary patterns of Australian adults and their association with socioeconomic status: results from the 1995 National Nutrition Survey. Eur J Clin Nutr 56, 687-693.

Montonen J, Knekt P, Harkanen T, Jarvinen R, Heliovaara M, Aromaa A \& Reunanen A (2005) Dietary patterns and the incidence of type 2 diabetes. Am J Epidemiol 161, 219-227.

Popkin BM (2003) Dynamics of the nutrition transition and its implications for the developing world. Forum Nutr 56, 262-264.

Ryan AS, Craig LD \& Finn SC (1992) Nutrient intakes and dietary patterns of older Americans: a national study. J Gerontol 47, M145-M150.

Shu XO, Gao YT, Yuan JM, Ziegler RG \& Brinton LA (1989) Dietary factors and epithelial ovarian cancer. Br J Cancer 59, 92-96.

Shu XO, Yang G, Jin F, Liu D, Kushi L, Wen W, Gao YT \& Zheng W (2004) Validity and reproducibility of the food frequency questionnaire used in the Shanghai Women's Health Study. Eur J Clin Nutr 58, 17-23.

Shu XO, Zheng W, Potischman N, Brinton LA, Hatch MC, Gao YT \& Fraumeni JF Jr (1993) A population-based case-control study of dietary factors and endometrial cancer in Shanghai. People's Republic of China. Am J Epidemiol 137, 155-165.

Smith CJ (1993) (Over)eating success: the health consequences of the restoration of capitalism in rural China. Soc Sci Med 37, 761-770.

Stampfer MJ, Hu FB, Manson JE, Rimm EB \& Willett WC (2000) Primary prevention of coronary heart disease in women through diet and lifestyle. $N$ Engl J Med 343, 16-22.

Subar AF, Thompson FE, Kipnis V, Midthune D, Hurwitz P, McNutt S, McIntosh A \& Rosenfeld S (2001) Comparative validation of the Block, Willett, and National Cancer Institute food frequency questionnaires: the Eating at America's Table Study. Am J Epidemiol 54, 1089-1099.

Wang GY \& Shen ZP, (1991) Chinese Food Composition Table, editors]. Beijing: People's Health Publishing House.

Wang W, Wu ZS, Zhao D, Wu GX, Wang WH, Liu J, Zeng ZC, Qin LP \& Liu J (2003) The trends of body mass index and overweight in population aged 25-64 in Beijing during 1984-1999. Zhonghua Liu Xing Bing Xue Za Zhi 24, 272-275.

Willett W (1998) Nutritional Epidemiology, 2nd ed. New York: Oxford University Press.

Woo J, Leung SS, Ho SC, Sham A, Lam TH \& Janus ED (1999) Influence of educational level and marital status on dietary intake, obesity and other cardiovascular risk factors in a Hong Kong Chinese population. Eur J Clin Nutr 53, 461-467.

World Health Organization (2006) WHO Collaborating Center on Surveillance of Cardiovascular Diseases. http://www.cvdinfobase. ca.

Wright AJ, Southon S, Bailey AL, Finglas PM, Maisey S \& Fulcher RA (1995) Nutrient intake and biochemical status of non-instutionalized elderly subjects in Norwich: comparison with younger adults and adolescents from the same general community. $\mathrm{Br} J$ Nutr 74, 453-475.

Wu G, Wu Z \& Liu J (2001) Trend of changes in mortality of cardiovascular diseases in some areas of Beijing during 1984 to 1998. Zhonghua Yu Fang Yi Xue Za Zhi 35, 98-101.

Yang L, Parkin DM, Li L \& Chen Y (2003) Time trends in cancer mortality in China: 1987-1999. Int J Cancer 106, 771-783.

Yu H, Harris RE, Gao YT, Gao R \& Wynder EL (1991) Comparative epidemiology of cancers of the colon, rectum, prostate and breast in Shanghai, China versus the United States. Int J Epidemiol 20, $76-81$.

Zheng W, Blot WJ, Shu XO, Gao YT, Ji BT, Ziegler RG \& Fraumeni JF Jr (1992) Diet and other risk factors for laryngeal cancer in Shanghai, China. Am J Epidemiol 36, 178-191.

Zheng W, Chow W, Yang G, et al. (2005) The Shanghai Women's Health Study: rationale, study design, and baseline characteristics. Am J Epidemiol 162, 1123-1131.

Zhou BF, Stamler J, Dennis B, Moag-Stahlberg A, Okuda N, Robertson C, Zhao L, Chan Q \& Elliott P (2003) INTERMAP Research Group. Nutrient intakes of middle-aged men and women in China, Japan, United Kingdom, and United States in the late 1990s: the INTERMAP study. J Hum Hypertens 17, $623-630$. 